\title{
Superadiabatic spin-preserving control of a single-spin qubit in a double quantum dot with spin-orbit interaction
}

\author{
Sergio S. Gomez and Rodolfo H. Romero* \\ Instituto de Modelado e Innovacion Tecnologica, \\ Universidad Nacional del Nordeste, CONICET, \\ Facultad de Ciencias Exactas y Naturales y Agrimensura, \\ Avenida Libertad 5400, W3404AAS Corrientes, Argentina
}

(Dated: October 31, 2019)

\begin{abstract}
A protocol for controlling the localization of an electron with a fixed projection of spin between two quantum dots in a material with spin-orbit $(\mathrm{SO})$ interaction is studied. Due to SO coupling, the manipulation of the electron shuttling between both quantum dots also leads to a mixing between spin projections near to the avoided crossing of levels. We use a transitionless quantum driving approach, with neglect of SO interaction, to analytically design simple electric and magnetic pulses able to rapidly drive the electron along an adiabatic Landau-Zener manifold. We show that the same fields in the presence of SO can also give a fast high-fidelity transition between the qubit states. The performance of the proposed protocol is assessed in the presence of SO interactions of typical semiconductor materials. Its is shown that it provides a fast and efficient spin-conserving method for controlling the electron position in a double quantum dot.
\end{abstract}

\section{INTRODUCTION}

Electrostatically defined quantum dot arrays in semiconductor materials are among of the most promising systems for the development of a technology leading to scalable quantum computers [1-7]. They are envisioned to take advantage of electron spin and orbital degrees of freedom for encoding information that have to be stored and transmitted throughout the device by applying controls consisting in time-dependent externally manipulated fields. Storage requires that a given state must be reliably preserved along time, while the processing operations demand a fast state transfer with high fidelity. All operations are assumed to be electrostatically controlled by external electrodes [8]. Physical effects on state storage and transfer can be studied in the simple system consisting in a single electron bond to two coupled quantum dots [9].

${ }^{*}$ Electronic address: rhromero@exa.unne.edu.ar 
The electron spin projection is a natural choice for encoding a qubit. They are usually distinguished by applying an external magnetic field splitting up and down energy levels. The presence of SO interaction in the used materials, although small, gives rise to experimentally observable mixing of spin states and allows for unwanted spin-flip processes [10]. The faster the change of the external potentials, the greater the state mixing. One way to reduce the mixing of states is by producing adiabatic (ideally infinitely slow) changes such that no transitions occur between up and down states. Nevertheless, such a process obviously spoils the processing speed of the device. Furthermore, other interactions and effects lead to decoherence in the state, thus diminishing the efficiency of the transfer. Several approaches have been advocated in order to speed up the adiabatic dynamics. They are generically named as shortcuts to adiabaticity, superadiabatic or transitionless quantum driving [11-13].

Adiabatic quantum computing is a procedure for solving optimization problems using the Schrödinger evolution of a quantum system. It consists in setting the system in the ground state of a Hamiltonian $H_{0}(t)$ at a given time $t=0$ and allows it to evolve adiabatically until reaching the ground state of a Hamiltonian $H(t)$, at a later time $t=T$, which encodes the solution of the problem. The assumption of an infinitely slow change guarantees reaching the required ground state of $H(T)$, where a measurement provides the sought solution [11, 14]. The approach termed transitionless quantum driving, aims to speed up the evolution along the adiabatic manifold within a finite time by introducing an additional perturbation $H_{I}(t)$ so that the state of the new Hamiltonian evolves along the adiabatic state of the original one. It has been applied to several problems such a chain of spins with a Heisenberg interaction [15], a spin under a time dependent magnetic field [12], and some particular cases of the three-level and four-level system with Landau-Zener potentials $[16,17]$.

In this work we apply the transitionless quantum driving approach to design a driving Hamiltonian speeding up the Landau-Zener (LZ) transfer of an electron between two quantum dots while still suppressing spin-flip processes due to spin-orbit interaction. We show that, for our model, the driving Hamiltonian derived in the absence of SO interaction, can be represented as a combination of electric and magnetic time-dependent pulses, whose duration and intensity is related to speed of the LZ process. Interestingly, the same pulses can also produce fast and high-fidelity transitions still in materials with strong SO interaction, which could provide an efficient manipulation in a realistic system.

The structure of this work is as follows. In Section II, we present the model Hamiltonian of the double quantum dot subjected to the time-dependent LZ process and a brief summary 


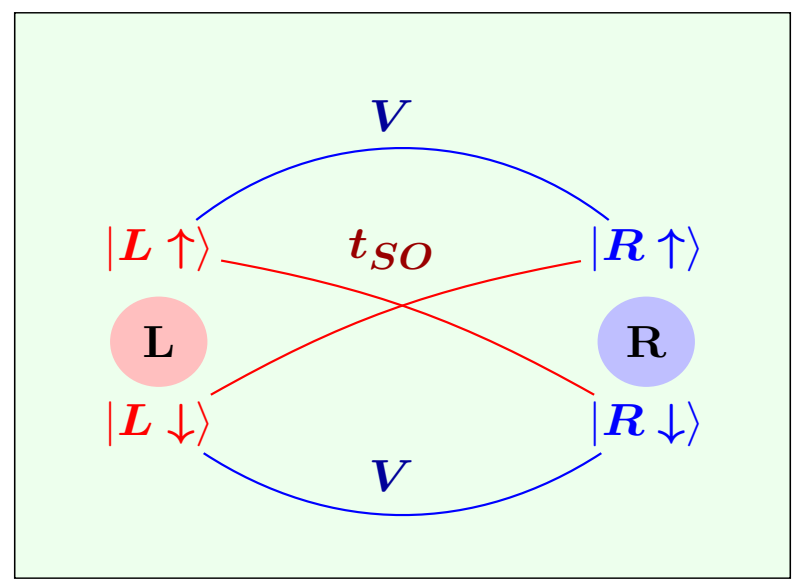

FIG. 1: Scheme of the system and its interactions. The circles represent the double quantum dot, labeled as left $(\mathrm{L})$ and right $(\mathrm{R})$. The lines depicts the transition amplitude between spin states of the same or opposite projections, i.e., spin-conserving $(V)$ and spin-flip $\left(t_{S O}\right)$ couplings.

of the transitionless quantum driving approach. Section III present the resulting quasi-adiabatic Hamiltonian and numerical results of the time-dependent probabilities of transition. Finally, in the last section some concluding remarks are given.

\section{THEORY}

\section{A. Landau-Zener processes in a double quantum dot with spin-orbit interaction}

We consider a single electron confined within a pair of coupled quantum dots ( $\mathrm{L}$ and $\mathrm{R}$ ) in a two-dimensional heterostructure taken as the $x y$ plane as depicted in Fig. 1 .

Both quantum dots are assumed identical to each other, so that their noninteracting ground state energies are taken as the energy offset of the system. Therefore, their energy levels are determined by the coupling between them, described by the Hamiltonian $H_{\text {hopping. A magnetic }}$ field $B_{z}$ perpendicular to the dots plane is also present, thus producing a Zeeman splitting of the levels $H_{\text {Zeeman. }}$ For most semiconductor materials, a non vanishing spin-orbit interaction $H_{S O}$ exists that mixes those magnetically-split levels. Furthermore, a time-dependent electric field interaction, $H_{\mathrm{LZ}}(t)$, is also applied for the purpose of controlling the electron state along a Landau-Zener process. Then, the whole system will be described by the Hamiltonian

$$
H_{0}=H_{\text {hopping }}+H_{\text {Zeeman }}+H_{\mathrm{SO}}+H_{\mathrm{LZ}}(t)
$$


which, using second quantization, will be phenomenologically described as

$$
\begin{aligned}
H_{\text {hopping }} & =-V \sum_{\sigma=\uparrow, \downarrow}\left(c_{\mathrm{L} \sigma}^{\dagger} c_{\mathrm{R} \sigma}+c_{\mathrm{R} \sigma}^{\dagger} c_{\mathrm{L} \sigma}\right) \\
H_{\text {Zeeman }} & =\Delta \sum_{k, \sigma} \sigma c_{k \sigma}^{\dagger} c_{k \sigma}+\text { h.c. } \\
H_{\mathrm{SO}} & =-t_{\mathrm{SO}} i \sum_{\sigma, \sigma^{\prime}}\left(\sigma_{y}\right)_{\sigma, \sigma^{\prime}} c_{R, \sigma}^{\dagger} c_{L \sigma^{\prime}}+\text { h.c. } \\
H_{\mathrm{LZ}}(t) & =\sum_{k, \sigma} \varepsilon_{k}(t) c_{k \sigma}^{\dagger} c_{k \sigma}+\text { h.c. }
\end{aligned}
$$

when expressed in the basis $|k, \sigma\rangle=c_{k, \sigma}^{\dagger}|v a c\rangle$, where $k=\mathrm{L}, \mathrm{R}$ is the state of the electron localized in the left or right quantum dots, and $\sigma=\uparrow, \downarrow$ is the $z$-projection of the electron spin. The parameter $\Delta=\hbar g \mu_{N} B_{z}$ is the Zeeman splitting due to the applied magnetic field $B_{z}, g$ being the Landé factor of the material and $\mu_{B}$ the Bohr magneton. The other parameters are a non-conserving spin hopping $t_{\mathrm{SO}}$, originated from the spin-orbit interaction associated with the Rashba mechanism [18], the spin-conserving hopping matrix element between the dots $V$ and the potential from the electric field applied along the interdot axis $\varepsilon_{i}(t)$. In the Landau Zener model, $\varepsilon_{i}(t)$ varies linearly with time. For our case, $\varepsilon_{L}(t)=\lambda t=-\varepsilon_{R}(t), \lambda$ being the speed of the change of the energy due to the electric field. The chosen values of the parameters $V, \Delta, t_{\mathrm{SO}}$ and $\lambda$ used in our calculations are derived from measurements in usual semiconductors and simple models [19-21]. So, for a tightbinding model, $t_{S O}=\alpha_{0} e E_{z} / 2 d$, where $d$ is the interdot distance, and $E_{z}$ the electric field in the perpendicular direction to the heterostructure plane. Choosing the Rashba constant $\alpha_{0}=5 \mathrm{~nm}^{2}$ as that for InSb, a semiconductor with one of the largest $\mathrm{SO}$ couplings, gives tipical $t_{S O} \simeq 0-500$ $\mu \mathrm{eV}$ and $V \simeq 10-1000 \mu \mathrm{eV}$ depending on the interdot distance. We have chosen $V=30 \mu \mathrm{eV}$, a typical $\lambda=5 \mathrm{meV} \mathrm{ns}^{-1}$ and $t_{S O} \simeq 0-60 \mu \mathrm{eV}$ in order to address the transition from weak to the strong spin-flip mechanism.

Fig. 2a shows the adiabatic levels for the Hamiltonian $H_{0}(t)$ as a function of time. At earlier times $(T \rightarrow-\infty)$, the ground state is $|L, \uparrow\rangle$ due to the large negative potential $\varepsilon_{\mathrm{L}}$ initially set at the dot L. At later times $(T \rightarrow \infty)$, the character of the ground state is mostly $|R, \uparrow\rangle$, acording to the inversion of the diabatic levels due to the LZ dependence. A sufficiently large Zeeman gap $\Delta>V$ clearly separates the states $|k, \uparrow\rangle$ from $|k, \downarrow\rangle(k=\mathrm{L}, \mathrm{R})$ for both dots. In the absence of SO effects, the spin projections are decoupled and become two independent two-level (L or R) systems. However, because of the finite SO coupling, there are avoided crossings between states of opposite spin projections at $t= \pm \Delta / 2 \lambda$, with a gap $\sim 2 t_{\mathrm{SO}}$. Also at $t=0$ there are two avoided crossings 
(a)

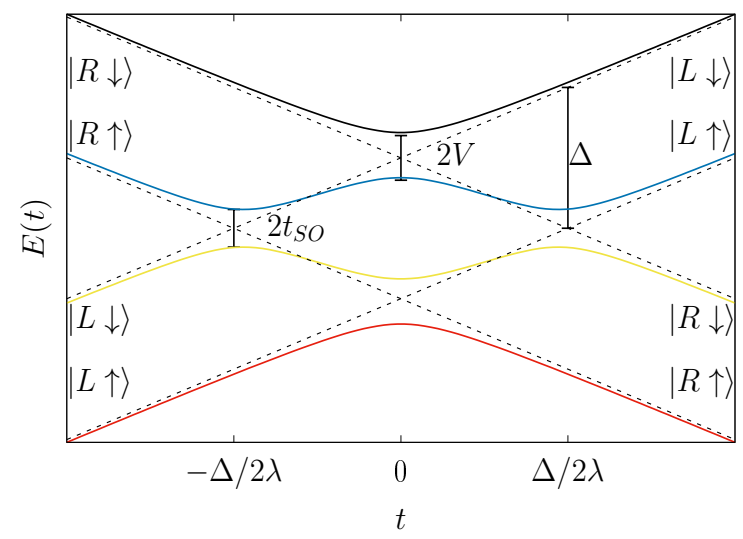

(b)

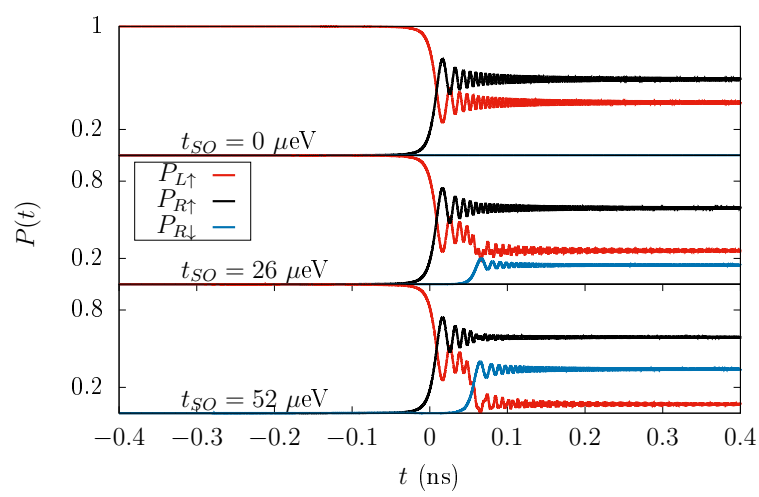

FIG. 2: (a) Adiabatic levels of the electron in the DQD as given by the Hamiltonian $H_{0}(t)$, as a function of time. The effect of the Zeeman coupling $(\Delta)$, interdot coupling $(V)$ and spin-orbit $\left(t_{\mathrm{SO}}\right)$ interaction on the splittings in the four-level spectrum are also shown. The dashed straight lines show the diabatic levels. (b) Dynamics of the probability of occupation of the states $|\mathrm{L} \uparrow\rangle$, $|\mathrm{R} \uparrow\rangle$ and $|\mathrm{R} \downarrow\rangle)$ as a function of time throughout a Landau-Zener process for $t_{S O}=0,26$ and 52 $\mu \mathrm{eV}$. As $t_{S O}$ increases, the probability of spin-flip transitions $|\mathrm{L} \uparrow\rangle \rightarrow|\mathrm{R} \downarrow\rangle$ becomes larger.

between levels of the same spin projection having a gap $\sim 2 V$. In particular, we are interested in the adiabatic process connecting the diabatic states $|\mathrm{L}, \uparrow\rangle$ and $|\mathrm{R}, \uparrow\rangle$, chosen as the qubits $|0\rangle$ and $|1\rangle$ of the computational space.

It should be noted from Fig. 2a that the state $\psi(t)$ evolves from $|\psi(-\infty)\rangle=|L, \uparrow\rangle$; hence, when the process is performed at a finite speed, after the first avoided crossing (at $t=0$ ) it can remain on the adiabatic level (thus performing to desired transition to $|\psi(t=\infty)=| R, \uparrow\rangle$ ), or jump to the first excited state (thus giving $|\psi(t=\infty)=| L, \uparrow\rangle$ in the long term). In the last case, there is still a probability to transition (at $t=\Delta /(2 \lambda))$ to the excited state leading to $|\psi(t=\infty)=| R, \downarrow\rangle$. Therefore, the three probabilities $P_{\mathrm{L}, \uparrow}(t), P_{\mathrm{R}, \uparrow}(t)$ and $P_{\mathrm{R}, \downarrow}(t)$ has to be considered, where $P_{\mathrm{R}, \downarrow}(t)$ must remain as small as possible because it represents a leakage from the computational space.

For two levels, the Landau Zener Formula accounts for the probability of the electron initially in the left site $\mathrm{L}$ at $t=-\infty$, after the evolution at $t=\infty$ transition to the site $\mathrm{R}$ [22-25]. This probability is $P_{R \uparrow}=1-\exp \left(-\pi / \xi_{\mathrm{LZ}}\right)$, where $\xi_{\mathrm{LZ}}=2 \hbar \lambda / V^{2}$ is a parameter that can be taken as the dimensionless speed of a Landau-Zener process between two states having an energy gap 
$\sim V$; for an infinitely slow adiabatic process $\left(\xi_{\mathrm{LZ}} \rightarrow 0\right)$ the probability $P_{R \uparrow}=1$, while it becomes smaller for fast LZ processes, such that $P_{R \uparrow} \rightarrow 0$ as $\xi_{\mathrm{LZ}} \rightarrow \infty$. As an example, typical values of experiments with InSb quantum dots, $V=30 \mu \mathrm{eV}$ and $\lambda=5 \mathrm{meV} \mathrm{ns}^{-1}$, give $P_{R \uparrow} \simeq 0.3$. When we switch on the spin-orbit interaction, the probability $P_{R \uparrow}$ remains approximately the same, but we observe an increase on $P_{R \downarrow}$. For a system having several avoided crossings, the probability for the Landau-Zener transition can be approximated as a sum of sequential two-level processes [26-31]. For the system considered here, $P_{R \downarrow} \simeq\left[1-\exp \left(-\pi / \xi_{\mathrm{SO}}\right)\right] \exp \left(-\pi / \xi_{\mathrm{LZ}}\right)$, where $\xi_{\mathrm{SO}}=2 \hbar \lambda / t_{\mathrm{SO}}^{2}$ is the dimensionless speed for the LZ transition throughout a gap $\sim t_{\mathrm{SO}}$ between the spin projections $\uparrow$ and $\downarrow$. The factors in $P_{R \downarrow}$ are the probabilities of the electron remaining in the same site after the first anticrossing and the probability of an spin-flip in the second anticrossing, respectively. In the Fig. $2 \mathrm{~b}$ the results of the evolution with the Hamiltonian $H_{0}(t)$ are shown. As mentioned above, as the $t_{S O}$ is increased from 0 to $V$, the probability of spin flip increases, reaching a value of approximately $15 \%$. On the other hand, the probability $P_{R \uparrow}$ also diminishes when $t_{S O}$ increases, due to the fact that the two-level LZ formula is not valid in this case. This is an indication of the effect of the spin orbit on the spin manipulation with the Landau-Zener protocol. The magnitude of the parameter $\lambda$ respect to both $V$ and $t_{S O}$ is relevant. Fast manipulation implies a significant lost of yield in the spin-conserving process $|\mathrm{L} \uparrow\rangle \rightarrow|\mathrm{R} \uparrow\rangle$.

\section{B. Transitionless quantum driving}

Landau-Zener manipulation, for given $V$ and $t_{\mathrm{SO}}$, can control the transfer of the state with high yields only for slow LZ processes $\left(\xi_{\mathrm{LZ}} \ll 1\right)$. We aim to use the transitionless quantum driving (TQD) method $[12,32,33]$ for improving the process speed while still maintaining high fidelities to the target state. In the TQD protocol a term, the superadiabatic correction $\hat{H}^{(1)}$, is added to the original Hamiltonian $\hat{H}_{0}$ to keep the evolution of the system along one of its adiabatic levels, defined as its instantaneous eigenstates

$$
\hat{H}_{0}(t)|n(t)\rangle=E_{n}(t)|n(t)\rangle .
$$

The adiabatic states could be assumed as solution of the time evolution of the Hamiltonian $\hat{H}_{0}$

$$
\left|\psi_{n}(t)\right\rangle=\exp \left(-\frac{i}{\hbar} \int_{0}^{t} E_{n}\left(t^{\prime}\right) d t^{\prime}-\int_{0}^{t}\left\langle n\left(t^{\prime}\right) \mid \partial_{t^{\prime}} n\left(t^{\prime}\right)\right\rangle\right)|n(t)\rangle
$$

However, the evolution will not, in general, follow an instant eigenvalue. By adding the control term $\hat{H}^{(1)}(t)$, the total Hamiltonian will follows the dynamics given by

$$
i \hbar \partial_{t}\left|\psi_{n}(t)\right\rangle=\left(\hat{H}_{0}(t)+\hat{H}^{(1)}(t)\right)\left|\psi_{n}(t)\right\rangle
$$


The correction $\hat{H}^{(1)}$ is obtained from the dynamics of the system

$$
i \hbar \partial_{t} \hat{U}(t)=\hat{H}(t) \hat{U}(t)
$$

from which the Hamiltonian can be expressed as

$$
\hat{H}(t)=\left(i \hbar \partial_{t} \hat{U}(t)\right) U^{\dagger}(t)
$$

in terms of the evolution operator $\hat{U}(t)$

$$
U(t)=\exp \left(-\frac{i}{\hbar} \int_{0}^{t} E_{n}\left(t^{\prime}\right) d t^{\prime}-\int_{0}^{t}\left\langle n\left(t^{\prime}\right) \mid \partial_{t^{\prime}} n\left(t^{\prime}\right)\right\rangle\right)|n(t)\rangle\langle n(0)| .
$$

Then, the superadiabatic correction results

$$
\hat{H}_{1}(t)=i \hbar\left(\sum_{n}\left|\partial_{t} n(t)\right\rangle\left\langle n(t)\left|-\left\langle n(t) \mid \partial_{t} n(t)\right\rangle\right| n(t)\right\rangle\langle n(t)|\right)
$$

or, equivalently,

$$
\hat{H}^{(1)}(t)=i \hbar \sum_{n \neq m} \frac{|n\rangle\left\langle n\left|\partial_{t} H_{0}\right| m\right\rangle\langle m|}{E_{m}-E_{n}} .
$$

We shall proceed now to obtain the explicit form of $\hat{H}^{(1)}$ for the Hamiltonian of the DQD, Eq. (1), such that its state evolves rapidly along adiabatic LZ states.

\section{SUPERADIABATIC LZ TRANSITIONS}

\section{A. Transitionless quantum driving approach}

We aim to use the approach presented above in order to speed up the switching between the qubit states along the adiabatic LZ ground state of Fig 2. Although a direct application of the superadiabatic approach is possible, it leads to a counterdiabatic control Hamiltonian, eq. (13), explicitly depending on the SO strength and, therefore, on the precise knowledge of the material properties. Instead, we shall firstly apply such an approach under the approximation of vanishing SO interaction and afterwards we will include its the effect by means of a physical approximation. Finally, we shall asses numerically the range of validity of such an approximation. As a result we will show that such a SO-independent control Hamiltonian still turns out to be highly efficient for a wide range of SO parameters of usually studied semiconductors.

In the absence of SO interaction, the Hamiltonian $H_{0}(t)$ is block diagonal and, therefore, $s_{z^{-}}$ conserving

$$
H_{s_{z}}^{(0)}(t)=\left(\begin{array}{cc}
\hat{h}_{-}^{(0)} & 0 \\
0 & \hat{h}_{+}^{(0)}
\end{array}\right)
$$


with

$$
\hat{h}_{ \pm}^{(0)}=\left(\begin{array}{cc} 
\pm \Delta+\varepsilon_{\mathrm{L}}(t) & -V \\
-V & \pm \Delta+\varepsilon_{\mathrm{R}}(t)
\end{array}\right)
$$

with $\varepsilon_{\mathrm{L} / \mathrm{R}}= \pm \lambda t$; the \pm sign labels each of the two $s_{z}= \pm \hbar / 2$ spin projections of the electron. Introducing the half-detuning $\varepsilon=\left(\varepsilon_{\mathrm{L}}-\varepsilon_{\mathrm{R}}\right) / 2$ and the average offset $\varepsilon_{a v}=\left(\varepsilon_{\mathrm{L}}+\varepsilon_{\mathrm{R}}\right) / 2$, it can be written as

$$
\hat{h}_{ \pm}^{(0)}(t)=\left(\begin{array}{cc} 
\pm \Delta(t)+\varepsilon(t) & -V \\
-V & \pm \Delta(t)-\varepsilon(t)
\end{array}\right),
$$

with $\Delta(t)=\Delta \pm \varepsilon_{a v}(t)$. For the usual LZ dependence, $\varepsilon_{a v}(t)=0$ and hence $\Delta(t)=\Delta$ becomes time-independent. For each one of these two-level Hamiltonian $\hat{h}_{ \pm}^{(0)}$, the counteradiabatic correction for the LZ protocol, $\varepsilon=\lambda t$, in the adiabatic basis is

$$
h^{(1)}(t)=i \hbar \partial_{t} \varepsilon\left(\begin{array}{cc}
0 & \frac{V}{2\left(\varepsilon^{2}+V^{2}\right)} \\
-\frac{V}{2\left(\varepsilon^{2}+V^{2}\right)} & 0
\end{array}\right)=V \vartheta\left(\xi_{\mathrm{LZ}}, t\right)\left(\begin{array}{cc}
0 & i \\
-i & 0
\end{array}\right),
$$

where the off-diagonal elements couple states localized at dots L and R, and

$$
\vartheta\left(\xi_{\mathrm{LZ}}, t\right)=\frac{\xi_{\mathrm{LZ}}}{\left(1+\xi_{\mathrm{LZ}}^{2} V^{2} t^{2} / \hbar^{2}\right)}
$$

describes the time-dependence of the interdot coupling required for a given finite-speed process $\xi_{\mathrm{LZ}}$; $h^{(1)}(t)$ vanishes for a infinitely slow LZ dynamics $\left(\xi_{\mathrm{LZ}}=0\right)$, but becomes $h_{\mathrm{LR}}^{(1)}(t) \simeq i \hbar^{2} / \xi_{\mathrm{LZ}} V t^{2} \sim$ $\mathcal{O}\left(t^{-2}\right)$ for large $\xi_{\mathrm{LZ}}$ values, so it becomes negligibly small, except within a time interval near to the avoided crossing occurring at $t=0$. Interestingly, this Hamiltonian depends on the departure from adiabaticity of the process, as measured by $\xi_{\mathrm{LZ}}$, and the interdot coupling $V$, but it does not on neither $\Delta(t)$ nor the initial spin projection.

Transforming to the diabatic basis set, the total Hamiltonian $\hat{H}_{s_{z}}=H_{s_{z}}^{(0)}(t)+\hat{H}_{s_{z}}^{(1)}(t)$ remains block diagonal

$$
\hat{H}_{s_{z}}=\left(\begin{array}{cc}
\hat{h}_{-} & 0 \\
0 & \hat{h}_{+}
\end{array}\right)=\left(\begin{array}{cc}
\hat{h}_{-}^{(0)}+\hat{h}^{(1)} & 0 \\
0 & \hat{h}_{+}^{(0)}+\hat{h}^{(1)}
\end{array}\right)
$$

where $\hat{h}_{ \pm}$take the form

$$
\hat{h}_{ \pm}=\left(\begin{array}{cc} 
\pm \Delta+\lambda t & -V(t) e^{i \Phi(t)} \\
-V(t) e^{-i \Phi(t)} & \pm \Delta-\lambda t
\end{array}\right)
$$

with 


$$
\begin{aligned}
\Phi(t) & =\tan ^{-1} \vartheta\left(\xi_{\mathrm{LZ}}, t\right) \\
V(t) & =V \sqrt{1+\vartheta^{2}\left(\xi_{\mathrm{LZ}}, t\right)} .
\end{aligned}
$$

Interestingly, the off-diagonal elements $\hat{h}_{\mathrm{LR}}$ of eq. (20) have amplitude $V(t)$ and a phase $\Phi(t)$. $V(t)$ is the bias associated to the electric field along the interdot axis. The phase $\Phi(t)$ formally reminds the form of Peierls substitution for a tight binding Hamiltonian subject to a magnetic field, i.e., $\hat{h}_{\mathrm{LR}} \rightarrow \hat{h}_{\mathrm{LR}} \exp (i \Phi)$ [34], where the magnetic field is incorporated into the phase of the hopping terms as the line integral of the vector potential $\mathbf{A}$ as $\Phi(t)=\int_{\mathrm{L}}^{\mathrm{R}} \mathbf{A}(\mathbf{r}, t) \cdot d \mathbf{l}$ to be consistent with the continuum description. Therefore, in our tight-binding model, we assign the origin of the time-dependent amplitude and phase in $\hat{h}_{\mathrm{LR}}$, to a time-dependent magnetic field $B_{z}(t)$ and a time dependent hopping $V(t)$. The corresponding diagonal Zeeman terms produced by such a $B_{z}(t)$ are absent due to it can be shown to lead to a vanishing counterdiabatic term, so that its only effect being the time-dependent phase modulation $\Phi(t)$. Assuming a uniform magnetic field $B_{z}(t)$ derived from a vector potential $\mathbf{A}(t)=\mathbf{B}(t) \times\left(\mathbf{r}-\mathbf{r}_{0}\right) / 2$, where $\mathbf{r}_{0}=\left(x_{0}, y_{0}\right)$ fixes the arbitrary gauge origin, the Peierls phase results $\Phi(t)=\int_{\mathrm{L}}^{\mathrm{R}} \mathbf{A}(\mathbf{r}, t) \cdot d \mathbf{l}=-B_{z}(t) y_{0} d$, i.e., proportional to the magnetic field $B_{z}(t)$ and the dot separation $d$, following the same time dependence as $\Phi(t)$, eq. (21), up to an arbitrary magnitude due to the freedom for the choice of the gauge origin.

Fig. 3 shows the form of the driving fields $\Phi(t)$ and $V(t)$, given by eqs. (21) and (22). Both of them are bell-shaped pulses amenable to experimental realization. They are centered around the time of the avoided crossing of the diabatic levels, have time widths $\tau_{\Phi}$ and $\tau_{V}$ (defined as half-distance between inflection points of the curves, as shown in Fig 3) and maximum values $\Phi_{\max }$ and $V_{\max }$. From eqs. (21) and (22), analytic expressions can be given for $\Phi_{\max }, \tau_{\Phi}$ and $V_{\max }$ (while $\tau_{V}$ has to be determined numerically), namely,

$$
\begin{aligned}
\Phi_{\max } & =\tan ^{-1} \xi_{\mathrm{LZ}} \\
\tau_{\Phi} & =\frac{2 \hbar}{\sqrt{3} V \xi_{\mathrm{LZ}}}\left(\sqrt{3 \xi_{\mathrm{LZ}}^{2}+4}-1\right)^{1 / 2} \\
V_{\max } & =V \sqrt{1+\xi_{\mathrm{LZ}}^{2}}
\end{aligned}
$$

For slow $\mathrm{LZ}$ processes $\left(\xi_{\mathrm{LZ}} \ll 1\right), \Phi_{\max } \simeq 0$ and the time-dependent driving interdot coupling $V(t)$ is rather flat and nearly time-independent $V(t) \simeq V$, except for times around $t=0$ where it presents a pronounced peak. As the speed $\xi_{L Z}$ of the LZ process increases, the pulses becomes stronger and tighter, i.e., $\Phi_{\max }$ and $V_{\max }$ increase while $\tau_{\Phi}$ and $\tau_{V}$ diminish. These pulses becomes 

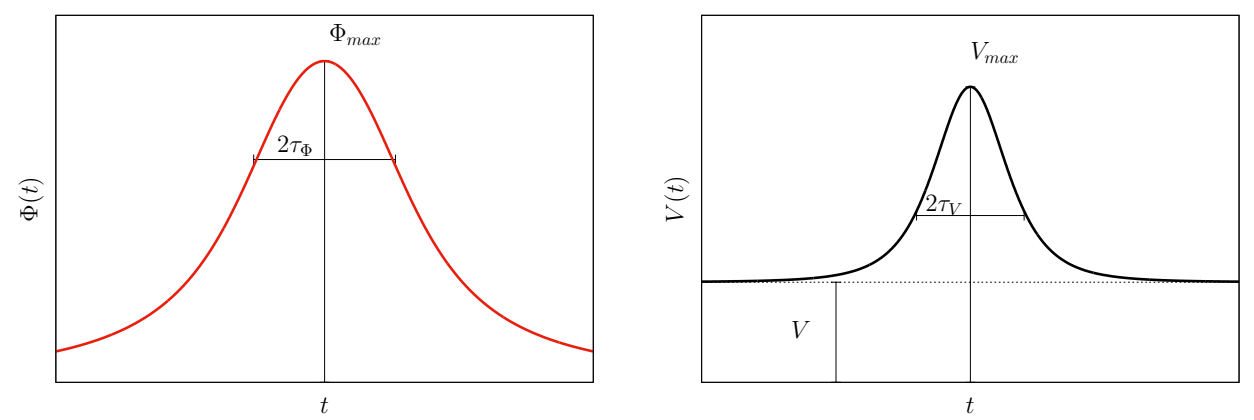

FIG. 3: Driving fields $\Phi(t)$ and $V(t)$, eqs. (21)-(22), calculated with the transitionless quantum driving approach aimed at following the adiabatic state of a LZ process. The pulses are asymptotically flat and act during a short time of order $\tau_{\Phi}$ and $\tau_{V}$.

particularly tighter and stronger for the fastest LZ processes as a direct consequence of the fact that the counterdiabatic fields must act strongly in shorter periods of time to hold the adiabaticity of fast LZ processes.

As a summary of the above discussion, we state that the counterdiabatic correction for our model, calculated within the transitionless quantum driving approach in the absence of SO effects, amounts to the simultaneous application of two time-dependent driving fields $B_{z}(t)$ and $V(t)$. From such a physical picture, we propose a dynamical control strategy using those same fields for the system with SO interaction. Since in that case, the dynamics will not be strictly adiabatic, we dubb it quasi-adiabatic (QA) as described by the Hamiltonian $H_{\mathrm{QA}}=H_{S_{z}}+H_{\mathrm{SO}}$

$$
\hat{H}_{\mathrm{QA}}(t)=\left(\begin{array}{cccc}
-\Delta^{\prime}(t)+\lambda t & -V(t) e^{i \Phi(t)} & 0 & t_{S O} e^{i \Phi(t)} \\
-V(t) e^{-i \Phi(t)} & -\Delta^{\prime}(t)-\lambda t & -t_{S O} e^{-i \Phi(t)} & 0 \\
0 & -t_{S O} e^{i \Phi(t)} & \Delta^{\prime}(t)+\lambda t & -V(t) e^{i \Phi(t)} \\
t_{S O} e^{-i \Phi(t)} & 0 & -V(t) e^{-i \Phi(t)} & \Delta^{\prime}(t)-\lambda t
\end{array}\right)
$$

where we have reintroduced the off-diagonal SO-dependent blocks, $\pm t_{\mathrm{SO}} \exp ( \pm i \Phi(t))$, mixing the up and down spin projections with their corresponding Peierls phases, and $\Delta^{\prime}(t)$ is the Zeeman splitting due to both the external field and $B_{z}^{\mathrm{QA}}(t)$. Now we shall numerically asses the efficiency of the Hamiltonian $H_{\mathrm{QA}}(t)$ for performing fast transitions between our qubit states.

\section{B. Quasi-adiabatic dynamics with SO interaction: numerical results}

Fig. 4 shows, in thick solid lines, the probability of occupation $P_{\mathrm{L} \uparrow}(t), P_{\mathrm{R} \uparrow}(t)$ and $P_{\mathrm{R} \downarrow}(t)$ as a function of time, calculated with $H_{\mathrm{QA}}(t)$ without SO interaction and with $t_{S O}=26 \mu \mathrm{eV}, 39$ 

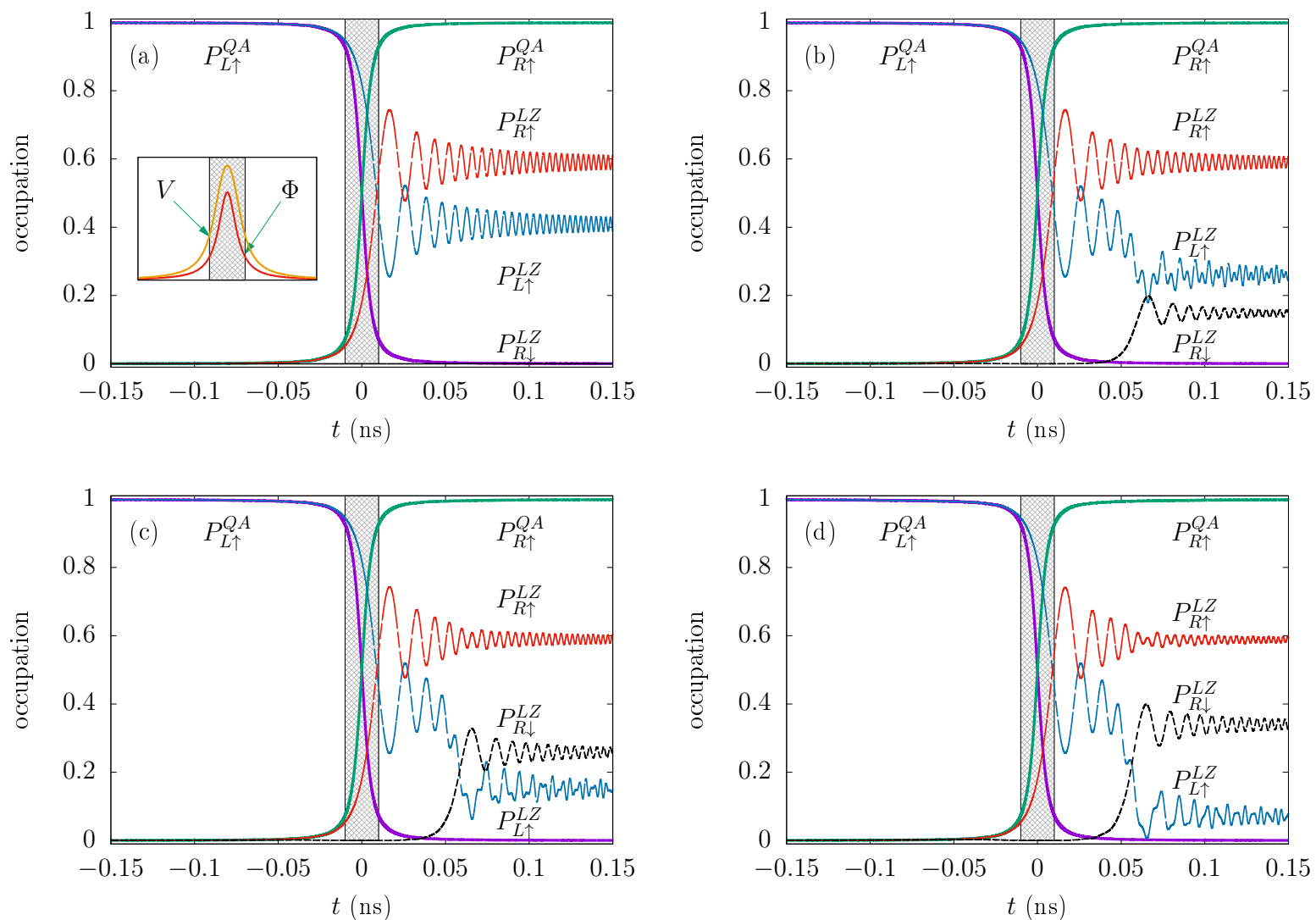

FIG. 4: Probabilities of occupation $P_{\mathrm{L} \uparrow}, P_{\mathrm{R} \uparrow}$ and $P_{\mathrm{R} \downarrow}$ calculated with the quasi-adiabatic approach (QA, solid lines) and Landau-Zener dynamics (LZ, dash-dotted lines), for $V=30 \mu \mathrm{eV}$, $\lambda=5 \mathrm{meV} \mathrm{ns}^{-1}$, with (a) $t_{\mathrm{SO}}=0 \mu \mathrm{eV}$, (b) $t_{\mathrm{SO}}=26 \mu \mathrm{eV}$, (c) $t_{\mathrm{SO}}=39 \mu \mathrm{eV}$ and (d) $t_{\mathrm{SO}}=52 \mu \mathrm{eV}$. In the first pannel we also show the corresponding pulses $V(t)$ (in units of $V_{\max }$ ) and $\Phi(t)$. The gray area highlight a time window $|t|<0.01 \mathrm{~ns}$ which approximately corresponds to both $\tau_{V}$ and $\tau_{\Phi}$

$\mu \mathrm{eV}$, and $52 \mu \mathrm{eV}$, hopping $V=30 \mu \mathrm{eV}$ and $\lambda=5 \mathrm{meV} \mathrm{ns}^{-1}$. For comparison, the Landau-Zener evolution is also shown in dash-dotted lines.

In the absence of SO effects (Fig. 4a), the QA dynamics is equivalent to the transitionless dynamics for the avoided crossing of the diabatic qubit states and produce a fast switching, achieving $P_{R \uparrow}^{\mathrm{QA}} \simeq 1$ within the range $|t| \lesssim 0.5$ ns. By contrast, the LZ process is not efficient for the desired spin-conserving transition $\mathrm{L} \rightarrow \mathrm{R}: P_{R \uparrow}^{\mathrm{LZ}} \simeq 0.6$ while $P_{L \uparrow}^{\mathrm{LZ}} \simeq 0.4 ; P_{R \downarrow}^{\mathrm{LZ}}=0$ since no transitions are allowed between different spin projections.

Figures $4 \mathrm{~b}, 4 \mathrm{c}$ and $4 \mathrm{~d}$ show the dynamics of the probabilities for increasing values of the SO parameter. The QA dynamics turns out quite robust as it remains similar to the case without SO; hence, the control with the counteradiabatic fields $B_{z}^{Q A}(t)$ and $V(t)$ becomes fast and efficient even 
for large values of $t_{\mathrm{SO}} \gtrsim V$. In all cases, the protocol holds a small leakage from the computational space, i.e., $P_{\mathrm{R} \downarrow}(t) \lesssim 10^{-3}$. By comparison, the LZ protocol spoils the transition as $t_{\mathrm{SO}}$ increases, enhancing the oscillations between the qubit states and the magnitude of the leakage. It should be noted that for the larger value $t_{\mathrm{SO}}=39 \mu \mathrm{eV}$, the $\mathrm{LZ}$ dynamics completely drives the system out of the computational space.

We study now the effect of interdot coupling $V$ on the fidelity of the transition performed with the QA Hamiltonian, eq. (26), for moderate and large SO parameters, as compared to LZ dynamics.
(a) Spin - conserving LZ
(b) Spin - conserving QA
$1-\mathrm{P}_{R \uparrow}$ $10^{5}\left(1-P_{R \uparrow}\right)$

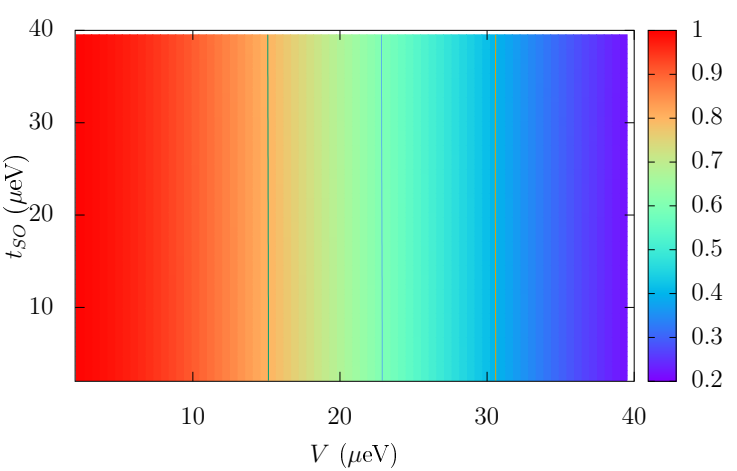

(c) Spin - flip LZ

$\mathrm{P}_{R \downarrow}$

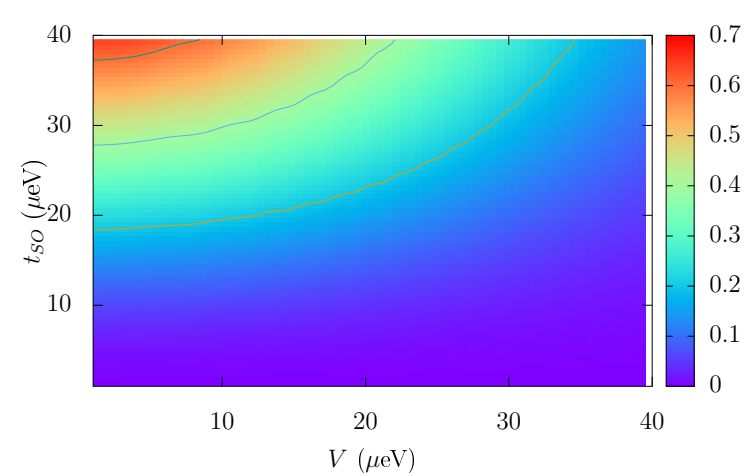

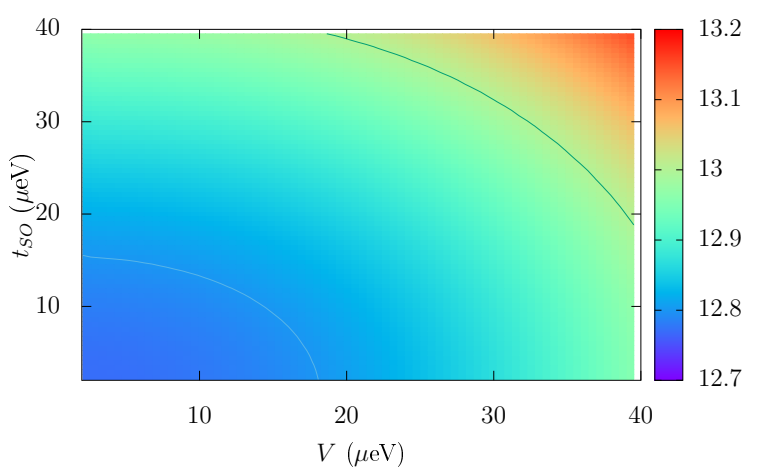

(d) Spin - flip QA $10^{3} \times P_{R \downarrow}$

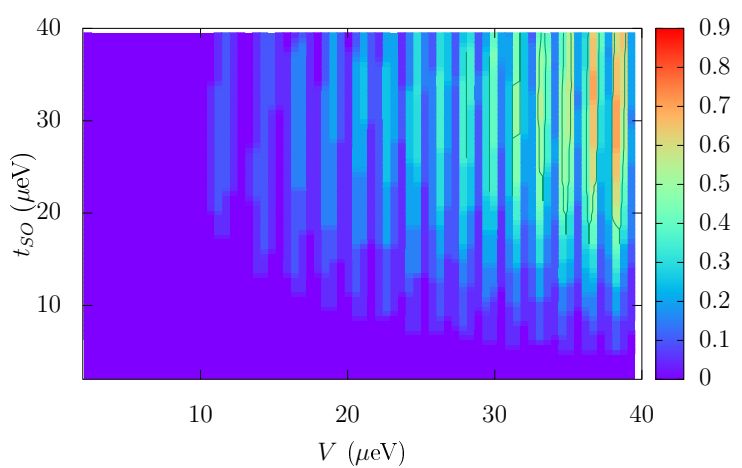

FIG. 5: Probability of electron transitions from the left to the right quantum dot for spin-conserving, $P_{R \uparrow}$ with LZ (a) and QA (b) strategies, and spin-flip, $P_{R \downarrow}$, processes (c) with Landau-Zener and QA dynamics(d), versus the spin-conserving hopping $V$ and the spin-flip hopping $t_{S O}$. 
In the Figure 5 we show a comparison between the spin-conserving and spin-flip probabilites for the LZ(pannels (a) and (c)) a QA strategies( pannels (b) and (d)) versus both the spin-conserving $V$ and spin-flip $t_{S O}$ hoppings. For the QA strategy, the spin conserving probability is very close to 1 , and therefore we have express it as $10^{5}\left(1-P_{R \uparrow}\right)$. On the other hand the spin flip probability is expressed as $10^{3} P_{R \downarrow}$, since its values are also very small. As expected, the $P_{R \uparrow}^{L Z}$ depends strongly on the values of $V$, roughly with the typical exponential behaviour given by the Landau Zener formulae, while is less sensitive to the value of $t_{S O}$. On the other hand, the spin-flip probability for LZ protocol is near 1 for small values of $V$ and large values of $t_{S O}$, following the approximation $P_{R \downarrow} \simeq\left[1-\exp \left(-\pi / \xi_{\mathrm{SO}}\right)\right] \exp \left(-\pi / \xi_{\mathrm{LZ}}\right)$, where $\xi_{\mathrm{SO}}=2 \hbar \lambda / t_{\mathrm{SO}}^{2}$. The QA strategy, on the other hand, increases the spin-conserving probability to a fidelity which differs from 1 in $\sim 10^{-3}$ for all of the values of $t_{S O}$ and $V$. On the other hand, the spin-flip transition probability is less than $0.1 \%$ for the all of the values of $V$ and $t_{S O}$, and therefore this shows the improvement that represents the use of the QA strategy respect to the LZ one.

\section{CONCLUSIONS}

We have studied the shuttling of an electron with a fixed projection of spin between two coupled quantum dots in the presence of the spin-orbit interaction. The electron states localized at left and right dot are assumed as suitable qubit states in charge qubits and can be connected by well-known LZ processes. We applied the transitionless quantum driving approach to produce a fast transition along the lowest adiabatic level of a given LZ process whithout SO Hamiltonian. The resulting time-dependent fields can be identified as a magnetic field $B_{z}^{\mathrm{QA}}(t)$ perpendicular to plane of the dots and an interdot coupling $V(t)$ driving the electron state along the adiabatic LZ level for each spin projection. The resulting driving fields are numerically tested in systems with SO coupling, such as InSb, using realistic parameters for those materials having strong SO effect. While LandauZener process become remarkably sensitive to the SO perturbation, with large leakage out of the computational space due to spin-flip transitions, the driving fields designed with the transitionles driving approach are almost SO-independent, giving rise to negligible leakage. We expect that this proposal can be useful for designing driving fields amenable of experimental realization and able to transfer rapidly the qubit states along a prescribed LZ adiabatic process while still protecting them from SO mixing. 


\section{ACKNOWLEDGMENTS}

This work was supported by Consejo Nacional de Investigaciones Científicas y Técnicas CONICET (Argentina) under grant PUE2017-22920170100089CO, and Universidad Nacional del Nordeste (Argentina) under grant PI17F020.

[1] S. A. Wolf, D. D. Awschalom, R. A. Buhrman, J. M. Daughton, S. von Molnár, M. L. Roukes, A. Y. Chtchelkanova, D. M. Treger, Science 294, 1488-1495 (2001).

[2] Žutić, I., Fabian, J. Sarma, S. D., Rev. Mod. Phys. 76, 323-386 (2004).

[3] Hanson, R., Kouwenhoven, L. P., Pette, J. R., Tarucha, S. Vandersypen, L. M. K. , Rev. Mod. Phys. 79, 1217-1261 (2007).

[4] Witzel, W. M. Sarma, S. D. , Phys. Rev. Lett. 98, 077601 (2007).

[5] Cywinski, L., Witzel, W. M. Sarma, S. D., Phys. Rev. Lett. 102, 057601 (2009).

[6] Hu, X. Sarma, S. D., Phys. Rev. Lett. 96, 100501 (2006).

[7] R. Li, L. Petit, D. P. Franke, J. P. Dehollain, J. Helsen, M. Steudtner, N. K. Thomas, Z. R. Yoscovits, K. J. Singh, S. Wehner, L. M. K. Vandersypen, J. S. Clarke, M. Veldhorst, Sci. Adv. 4, eaar3960 (2018).

[8] X. Li, E. Barnes, J. P. Kestner, and S. Das Sarma, Phys. Rev. A 96, 012309 (2017).

[9] M. J. Rancic and D. Stepanenko, Phys. Rev. B 94, 241301 (2016).

[10] Zhi-Hai Liu, R. Li, X. Hu and J. Q. You, Sci. Rep. 8, 2302 (2018).

[11] K. Takahashi, arXiv:quant-ph/1808.07177.

[12] M. V. Berry, J. Phys. A 42, 365303 (2009).

[13] E. Torrontegui, S. Ibáñez, S. Martínez-Garaot, M. Modugno, A. del Campo, D. Guéry-Odelin, A. Ruschhaupt, X. Chen, and J. G. Muga, Adv. in At., Mol., and Opt. Phys. 62, 117 (2013).

[14] T. Albash, D. A. Lidar, Rev. Mod. Phys. 90, 015002 (2018).

[15] Bi-Hua Huang, Yi-Hao Kang, Ye-Hong Chen, Zhi-Cheng Shi, Jie Song, and Yan Xia, Phys. Rev. A 97, 012333 (2018).

[16] L. Giannelli and E. Arimondo, Phys. Rev. A 89, 033419 (2014).

[17] M. Theisen, F. Petiziol, S. Carretta, P. Santini, and S. Wimberger, Phys. Rev. A 96, 013431 (2017)

[18] Francisco Mireles and George Kirczenow, Phys Rev. B, 64, 024426 (2001)

[19] G. Burkard, D. Loss, and D. P. DiVincenzo, Phys. Rev. B 59, 2070 (1999).

[20] O. Voskoboynikov, C. P. Lee, and O. Tretyak, Phys. Rev. B 63 , 165306 (2001); C. F. Destefani, S. E. Ulloa, and G. E. Marques, ibid. 69, 125302 (2004).

[21] J. B. Miller, D. M. Zumbühl, C. M. Marcus, Y. B. Lyanda-Geller, D. Goldhaber-Gordon, K. Campman, and A. C. Gossard Phys. Rev. Lett. 90, 076807(2003).

[22] L. Landau, Phys. Z. Sowjetunion 2, 46 (1932). 
[23] C. Zener, Proc. R. Soc. London, Ser. A 137, 696 (1932).

[24] E. C. G. Stueckelberg, Helv. Phys. Acta (Basel) 5, 369 (1932).

[25] E. Majorana, Il Nuovo Cimento (1924-1942) 9, 43 (1932).

[26] Y. N. Demkov and V. Osherov, JETP 26, 916 (1968). [25] C. Carroll and F. Hioe, J. Phys. A: Math. Gen. 19, 2061 (1986).

[27] Y. Kayanuma and S. Fukuchi, J. Phys. B: At. Mol. Phys. 18, 4089 (1985).

[28] V. L. Pokrovsky and N. A. Sinitsyn, Phys. Rev. B 65, 153105 (2002).

[29] Y. N. Demkov and V. Ostrovsky, J. Phys. B 34, 2419 (2001).

[30] B. Damski, Phys. Rev. Lett. 95, 035701 (2005).

[31] A. A. Rangelov, J. Piilo, and N. V. Vitanov, Phys. Rev. A 72, 053404 (2005).

[32] M. G. Bason, M. Viteau, N. Malossi, P. Huillery, E. Arimondo, D. Ciampini, R. Fazio, V. Giovannetti, R. Mannella, and O. Morsch, Nat. Phys. 8, 147 (2012).

[33] M. Demirplak and S. A. Rice, J. Phys. Chem. A 107, 9937 (2003).

[34] R. Peierls, Z. Phys. 80, 763-791 (1933). doi:10.1007/bf01342591. 